\title{
M87o-Transduced CD34+ Peripheral Blood Stem Cells
}

National Cancer Institute

\section{Source}

National Cancer Institute. M870-Transduced CD34+ Peripheral Blood Stem Cells. NCI

Thesaurus. Code C82351.

Peripheral blood stem cells (PBSCS) transduced with the retroviral vector M87o encoding for the HIV-1-entry inhibitor peptide membrane-anchored antiviral peptide C46 (maC46). Expression of C46 by M87o-transduced CD34+ peripheral blood stem cells may prevent the fusion of viral and cellular membranes, thereby inhibiting HIV-1 entry. C46 is a membrane-anchored peptide encoding amino acids 628 to 673 of the HIV-1 entry inhibitory transmembrane glycoprotein gp41. 